Pacific Journal of Mathematics

MINIMAL SURFACES AND HEEGAARD SPLITTINGS OF THE 


\title{
MINIMAL SURFACES AND HEEGAARD SPLITTINGS OF THE THREE-TORUS
}

\author{
Charles Frohman
}

\begin{abstract}
An unknotting criterion for proper arcs in $F \times I$ is proved. This is then applied to show the topological uniqueness of least genus one-sided Heegaard splittings of the three-torus and of genus three two-sided minimal surfaces in a flat three-torus.
\end{abstract}

Lawson has proved that if $F_{1}, F_{2} \subseteq S^{3}$ are embedded minimal surfaces having the same genus in metrics having positive Ricci curvature, then there exists a homeomorphism $h: S^{3} \rightarrow S^{3}$ with $h\left(F_{1}\right)=F_{2}$. Meeks has proved that up to homeomorphism there is at most one minimal surface $F$ of genus $g$ in a flat, convex three-ball $B$ having a given Jordan curve $\gamma \subseteq \partial B$ as boundary. The outlines of their respective arguments are the same. First it is shown that the minimal surface of interest is a Heegaard splitting, then the topological uniqueness of Heegaard splittings of the three-sphere is used to derive the result. In this paper we prove that up to homeomorphism there is only one surface of genus 3 in the three-torus that can be a minimal surface in a flat metric on the three-torus. The outline of the proof is similar to the proofs of Lawson and Meeks described above, except that because so little is known about the Heegaard splittings of the three-torus, the topological part of our argument is more involved than theirs.

The notion of a one-sided Heegaard splitting was introduced by Rubinstein in [R]. In this paper we prove that all least genus, one-sided Heegaard splittings of the three-torus are topologically equivalent. This result and our result concerning genus three minimal surfaces are both proved using the following unknotting lemma. We say a proper arc in $F \times I$ is unknotted if it is isotopic to $* \times I$.

LEMMA 1.1. Let $F$ be a closed surface of positive genus, and $k$ a proper arc in $F \times I$. The arc $k$ is unknotted if and only if ${ }^{-}(F \times I-N(k))$ is a handlebody.

This lemma generalizes Papakyriakopoulos' unknotting lemma for circles in the three-sphere. In the early 1970's, E. Brown [B] and C. 
Feustal, $[\mathbf{F e}]$, developed results that are similar to Lemma 1.1. Although it is enticing to try to prove our result directly from [Fe], it is not clear that there is any savings in doing so.

We will assume that all spaces have a fixed PL structure and that all maps are PL. Furthermore we assume that the reader is familiar with the theory of incompressible surfaces and Haken manifolds as is described in [W2, W3]. A Heegaard splitting of a closed three-manifold is a surface $F \subseteq M$ such that $M-F$ is the union of two open handlebodies. A one-sided Heegaard splitting is a surface $K \subseteq M$ such that $M-K$ is an open handlebody. We say that two (one-sided) Heegaard splittings $F$ and $F^{\prime}$ of $M$ and $M^{\prime}$ are topologically equivalent if there exists a homeomorphism $h: M \rightarrow M^{\prime}$ such that $h(F)=F^{\prime}$. We say that two subsets $X, X^{\prime}$ of $M$ are isotopic if there exists an isotopy $h: M \times I \rightarrow M$ such that $h_{0}=$ Id and $h_{1}(X)=X^{\prime}$. We say that a (one-sided) Heegaard splitting $F$ has least genus if the genus of $F$ is minimal among all (one-sided) Heegaard splittings of $M$.

1. The unknotting lemma. The purpose of this section is to prove the following lemma.

LEMMA 1.1. Let $F$ be a closed surface of positive genus, and $k$ a proper arc in $F \times I$. The arc $k$ is unknotted if and only if ${ }^{-}(F \times I-N(k))$ is a handlebody.

An easy corollary of this is:

COROllary 1.2. Let $F$ be a closed surface of positive genus, and $k$ a proper arc in $F \times I$. The arc $k$ is unknotted if and only if $\pi_{1}(F \times I-N(k), *)$ is free.

Remark. When $F$ is homeomorphic to $S^{2}$ the lemma is false. For $F$ homeomorphic to $S^{1} \times S^{1}$ we have an algebraic proof based on the fact that one-relator presentations of $\pi_{1}\left(S^{1} \times S^{1}, *\right)$ are "standard". The idea for the proof we give here is due to Marty Scharlemann.

Proof. First assume that $k$ is a proper arc in $F \times I$ that is isotopic to $* \times I$. We then know that ${ }^{-}(F \times I-N(k))$ is homeomorphic to - $((F-N(*)) \times I)$ where $N(*)$ is a regular neighborhood of $*$ in $F$. Let $p:{ }^{-}(F-N(*)) \times I \rightarrow^{-}(F-N(*))$ be the natural projection. 
Choose a family of nontrivial arcs $\left\{c_{i}\right\}$ on ${ }^{-}(F-N(*))$ that are pairwise disjoint and cut ${ }^{-}(F-N(*))$ into a disk. Notice that $\left\{p^{-1}\left(c_{i}\right)\right\}$ is a system of meridian disks cutting ${ }^{-}((F-N(*)) \times I)$ into a ball. Therefore ${ }^{-}(F \times I-N(k))$ is a handlebody.

Now assume that ${ }^{-}(F \times I-N(k))$ is a handlebody. Let $M$ be a meridian disk for ${ }^{-}(F \times I-N(k))$ such that $\partial M$ is transverse to fr $N(k)$ in $\partial^{-}(F \times I-N(k))$ and the number of components of $\partial M \cap \operatorname{fr} N(k)$ is minimal. Since $F \times\{i\} \subseteq F \times I, i \in\{0,1\}$, is incompressible we see that $-(F \times\{i\}-N(k))$ is an incompressible surface in $-(F \times I-N(k))$. Thus the number of components of $\partial M \cap \operatorname{fr} N(k)$ is at least 2. The regular neighborhood $N(k)$ supports a product structure $k \times D^{2}$ where $k$ is the set $k \times\{0\}$. Since $\partial M \cap \operatorname{fr} N(k)$ has the minimum number of components we may assume that in our coordinate system $\partial M \cap \operatorname{fr} N(k)$ $=k \times\left\{d_{i}\right\}_{i=1}^{n}$, where $d_{i} \in \partial D^{2}$. Viewing $D^{2}$ as the cone of $\partial D^{2}$ on 0 we construct $\bar{M}$ by letting

$$
\bar{M}=M \cup\left\{\left(\rho d_{i}, t\right) \in N(k) \mid \rho, t \in[0,1]\right\} .
$$

We can view $\bar{M}$ as a disk $\bar{D}$ with segments of its boundary identified. In specific we may partition the boundary into closed segments $\left\{b_{i}\right\}_{i=1}^{n}$ and open segments $\left\{a_{i}\right\}_{i=1}^{n}$ such that the segments $b_{i}$ are identified to one another (and mapped homeomorphically onto $k$ ) and the segments $a_{i}$ are disjoint from one another and lie in $F \times\{0,1\}$. See Figure 1 for a schematic description. Throughout this argument when we want to view a

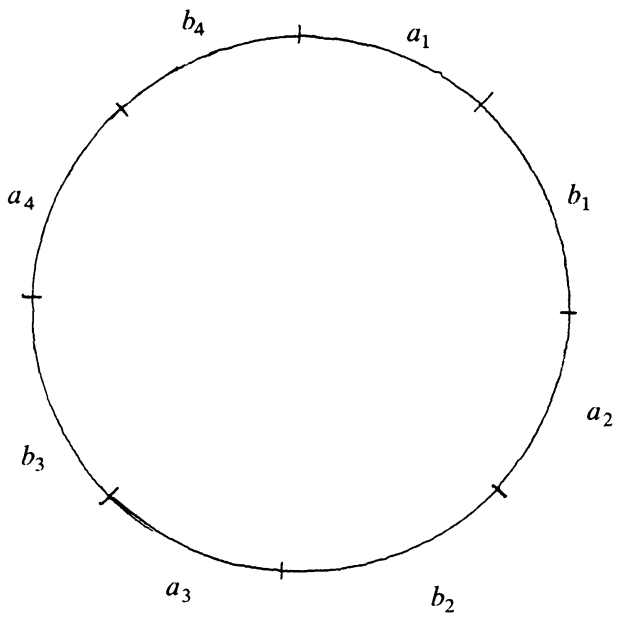

FIGURE 1 
subset of $\bar{M}$ as being in $F \times I$ we will refer to it as contained in $\bar{M}$. Because of the inductive nature of our argument we need to view subsets of $\bar{M}$ as subsets of $\bar{D}$, in these cases we will refer to them as subsets of $\bar{D}$.

Let $p: F \times I \rightarrow F$ be the canonical projection. A subset $S$ of $F \times I$ is vertical if $p^{-1}(p(S))=S$. Let $A, V_{1}, \ldots, V_{m}$ be a system of vertical surfaces in $F \times I$ such that $A$ is a proper annulus, $\partial V_{i} \subseteq F \times\{0,1\} \cup A$ and ${ }^{-}\left(F \times I-N\left(A \cup \cup_{i} V_{i}\right)\right)$ is a three-ball. Furthermore we may assume that $\partial k \cap\left(A \cup \cup_{i} V_{i}\right)=\varnothing, \bar{M}$ is transverse to $A, \bar{D} \cap A$ consists of only arcs that are proper in $\bar{D}$, and finally the number of components of $\bar{D} \cap A$ is minimal among all compressing disks $\bar{D}$.

If there exists an arc $\chi$ in $\bar{D} \cap A$ or $\bar{D} \cap V_{i}$ such that the endpoints of $\chi$ lie in distinct intervals $a_{i}$ and $a_{j}$ of $\partial \bar{D}$ with $a_{i}$ and $a_{j}$ being adjacent to an interval $b_{l}$, then the disk $D^{\prime} \subseteq \bar{D}$ cut out by $\chi$ and containing $b_{l}$ can be used to isotope $k$ into $A$ or $V_{i}$, (see Figure 2). This is enough to show that $k$ is unknotted. Consequently we will call an arc $\chi$ as described above an excellent arc. The proof of Lemma 1.1 consists of a step by step search for an excellent arc in $\bar{D} \cap A$ or $\bar{D} \cap V_{i}$ for some $i$. If there is no excellent arc in $\bar{D} \cap\left(A \cap V_{1} \cdots \cup V_{l}\right)$ then we will isotope $\bar{D}$ so that it is in a "nice" position and continue our search in $\bar{D} \cap V_{i+1}$. If we get all the way through $\bar{D} \cap\left(A \cup \bigcup_{i} V_{l}\right)$ without finding an excellent arc then we derive a contradiction.

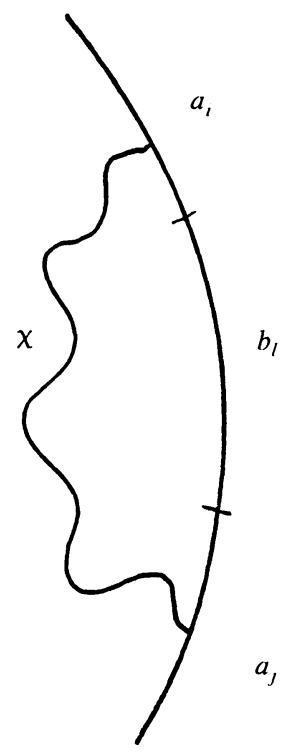

FIGURE 2 
Step 1. Assume that there are no excellent arcs in $\bar{D} \cap A$. We will isotope $\bar{D}$ so that $\bar{D} \cap A$ consists only of vertical arcs missing $\cup V_{i}$. We distinguish five different types of arcs in $\bar{D} \cap A$. The first and second types have their endpoints in $\bigcup b_{i}$. An arc of the third type has its endpoints in some $a_{i}$. An arc of the fourth type has one endpoint in some $a_{i}$ and the other in some $b_{j}$. Finally the fifth type of arc has its endpoints in distinct intervals $a_{l}$ and $a_{J}$. If $\chi$ is an arc in $\bar{D} \cap A$ of the first four types that is outermost on $\bar{D}$ then we know how to isotope $\bar{M}$ so that $\bar{D} \cap A$ is simplified. If there are no outermost arcs $\chi$ of types one through four, then every arc in $\bar{D} \cap A$ is of type five. We show that if this is the case, then $\bar{M}$ can be isotoped so that $\bar{M} \cap A$ is vertical and misses $\cup V_{l}$. This will complete step one of the proof.

Assume that $\chi$ is an arc in $\bar{D} \cap A$ that is outermost on $\bar{D}$, and of the first four types. There are four cases to consider.

Case 1. The endpoints of $\chi$ lie in some $b_{i}$. Let $D^{\prime}$ be the subdisk of $\bar{D}$ cut out by $\chi$ that intersects $A$ only in $\chi$. Since no pairs of distinct points of $b_{l}$ are identified to one another in forming $\bar{M}$, the disk $D^{\prime}$ is embedded in $F \times I$. Use this disk as a guide to isotope $\bar{M}$ so that there are two fewer points of intersection in $k \cap A$. Then isotope $\bar{M}$ relative to $k$ to remove any simple closed curves that may have been created in $A \cap \bar{D}$.

Case 2. The endpoints of $\chi$ lie in distinct intervals $b_{i}$ and $b_{j}$. Since if $b_{i} \cap A=\varnothing$ for any $i$ then $b_{i} \cap A=\varnothing$ for all $i$, we may assume that the intervals $b_{i}$ and $b_{j}$ are adjacent on $\partial \bar{D}$, with some $a_{k}$ between them.

Since $\chi$ is outermost the endpoints of $\chi$ must be identified in $\bar{M}$. There are two situations. The first is when $\chi$ forms a trivial simple closed curve on $A$, and the second when $\chi$ forms a nontrivial simple closed curve on $A$. For illustrations of these situations see Figure 3.

Situation a. In this case $\chi$ bounds a disk $D^{\prime \prime}$ on $A$ and $D^{\prime \prime} \cup D^{\prime}$ form a disk in $F \times I$ having $\bar{a}_{k}$ as its boundary. Since $\partial(F \times I)$ is incompressible the simple closed curve $\bar{a}_{k}$ bounds a disk on $\partial(F \times I)$. We may use this disk to isotope $M$ so that $\partial M \cap \operatorname{fr} N(k)$ has fewer components. Hence situation a cannot occur.

Situation b. Since the fundamental group of $F \times I$ is carried by either of its boundary components, we have that $\bar{a}_{k}$ and one of the boundary components $B$ of $A$ bound an annulus on $\partial(F \times I)$. Furthermore $D^{\prime}$ is identified to itself along $D^{\prime} \cap b_{i}$ and $D^{\prime} \cap b_{j}$ to form an annulus, and $B$ 


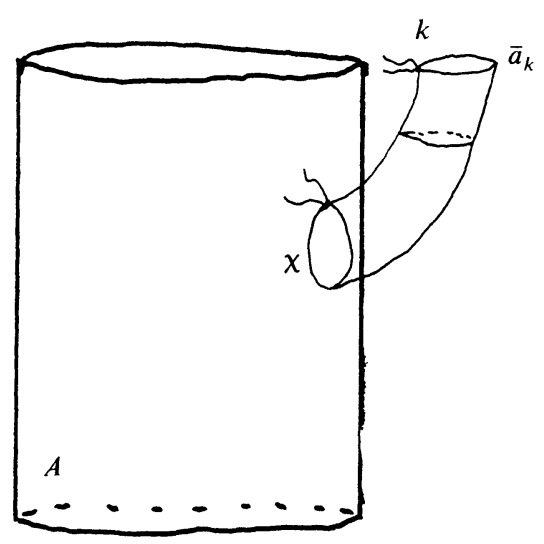

a

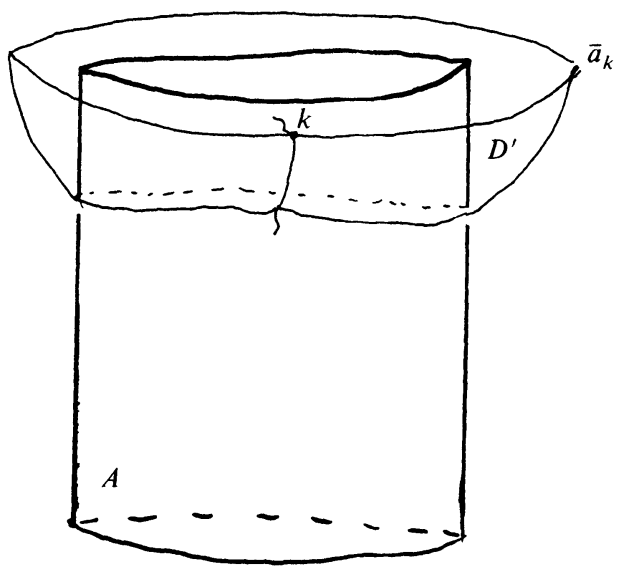

$\mathrm{b}$

FigURE 3

and $\chi$ bound an annulus on $A$. These three annulli form the boundary of a solid torus in $F \times I$ that can be used as a guide to isotope $\bar{M}$ so as to reduce the cardinality of $k \cap A$.

Case 3. Suppose $\chi$ has both its endpoints in the same $a_{i}$. Since $\partial(F \times I)$ is incompressible some arc of $a_{i}$ along with an arc of $\partial A$ bound a disk in $\partial(F \times I)$, this along with the disks on $A$ and $\bar{D}$ split off by $\chi$ bound a ball. Use this as a guide to isotope $\bar{M}$ so as to reduce the number of components of $\bar{D} \cap A$.

Case 4. One endpoint of $\chi$ lies in an interval $a_{i}$, and the other endpoint of $\chi$ lies in an interval $b_{j}$. Since $\chi$ is outermost the arcs $a_{i}$ and $b_{j}$ must be adjacent on $\partial \bar{D}$. Hence the outermost disk $D^{\prime}$ cut out by $\chi$ has boundary consisting of $\chi$, a segment of $b_{j}$ and a segment of $a_{i}$. Use $D^{\prime}$ as a guide to isotope $\bar{M}$ so as to reduce the cardinality of $k \cap A$. Then isotope away any simple closed curves in $\bar{D} \cap A$ that may have been created.

Because of the constructions above we may assume that if $\chi$ is an outermost arc in $\bar{D} \cap A$, then the endpoints of $\chi$ lie in distinct intervals $a_{\imath}$ and $a_{j}$. Let $D^{\prime} \subseteq \bar{D}$ be an outermost disk cut out by $\chi$. Since the intervals $a_{i}$ and $b_{l}$ alternate on $\partial \bar{D}$ there must be some interval $b_{l}$ in $\partial D^{\prime}$. But this means that $b_{l} \cap A=\varnothing$. Since the intervals $\bigcup b_{i}$ are all identified to one another in $\bar{M}$ we have that there are no $\operatorname{arcs}$ in $\bar{D} \cap A$ having an endpoint in $\bigcup b_{i}$. If there exists an arc in $\bar{D} \cap A$ both of whose endpoints 
in an interval $a_{i}$, then there exists an outermost such arc. Hence our assumption that all the outermost arcs in $\bar{D} \cap A$ are of type five implies that all the arcs in $\bar{D} \cap A$ are of the fifth type. Without looking further we can distinguish two types of such arcs. An arc of type $i$ has both its endpoints in the same component of $\partial(F \times I)$. An arc of type ii has its endpoints in distinct components of $\partial(F \times I)$.

Type i. Let $D^{\prime}$ be the disk on $A$ that is cut out by $\chi$. By passing to a different $\chi$ if necessary we may assume that int $D^{\prime} \cap \bar{M}=\varnothing$. Replace one of the components of $\bar{D}-\chi$ by a pushoff of $D^{\prime}$, call the new singular disk $\bar{M}^{\prime}$. Notice that the number of components of $\bar{M}^{\prime} \cap \operatorname{fr} N(k)$ is smaller than the number of components of $\bar{M} \cap \operatorname{fr} N(k)$. This contradicts our choice of $\bar{M}$.

Type ii. We may thus assume that all $\chi$ are of type ii. Isotope $\bar{M}$ so that $\chi$ is vertical in $A$ and $\chi \cap\left(\cup V_{i}\right)=\varnothing$ without increasing the number of components of $\bar{D} \cap A$.

We may thus assume that $k \cap A=\varnothing$ and $\bar{M} \cap A$ consists of vertical arcs that miss $\cup V_{l}$.

Step 2. Assume that if $i<r$ then $\bar{M} \cap V_{l}$ and $\bar{M} \cap A$ consists of vertical arcs that miss the vertical edges of $\cup V_{i}$. Furthermore assume that there are no excellent arcs in $\bar{D} \cap\left(A \cup \cup_{i<r} V_{l}\right)$. Isotope $\bar{M}$ relative to $A \cup V_{1} \cdots \cup V_{r-1}$ so that $\bar{M}$ is transverse to $V_{r}$ and the number of components of $\bar{M} \cap V_{r}$ is minimal. It is possible to remove and straighten arcs of intersection in $\bar{D} \cap V_{r}$ as we did with the annulus. It is worth noting that the arcs in Case 2, situation b cannot occur. All the arguments in the other cases still go through. If there are no excellent arcs in $\bar{D} \cap V_{r}$ continue the process for $V_{r+1}$.

Suppose that after performing our normalization process for all $V_{i}$ that there are no excellent $\operatorname{arcs}$ in $\bar{D} \cap\left(A \cup \cup_{l} V_{l}\right)$. If we cut $F \times I$ along $A \cup \cup_{i} V_{l}$ we get a manifold $\overline{F \times I}$ that is homeomorphic to $D^{2} \times I$ in such a way that $\partial D^{2} \times I$ is the image of the frontier of a regular neighborhood $N\left(A \cup \cup_{i} V_{i}\right)$, and the images of the $\operatorname{arcs} \bar{M}$ $\cap \partial N\left(A \cup \cup_{i} V_{i}\right)$ are vertical. Let $\tilde{M}$ denote the image of $\bar{M}$ in $\overline{F \times I}$. Since $k$ is disjoint from $A \cup \bigcup_{i} V_{i}$ we have that $\tilde{M}$ consists of one component $\tilde{M}^{\prime}$ that is a singular disk, and some components that are properly embedded disks. Notice that $\partial^{-}(\widetilde{F \times I}-N(k))$ is a torus and each component of ${ }^{-}\left(\tilde{M}^{\prime}-N(k)\right)$ is a compressing disk. Hence $-(\widetilde{F \times I}-N(k))$ is a solid torus. Notice that returning $N(k)$ to 
- $(\overline{F \times I}-N(k))$ is the same as glueing a plate to the solid torus to obtain a three ball. Hence if $w$ is a nontrivial simple closed curve on $\operatorname{fr} N(k)$ then $w$ has algebraic intersection $\pm I$ with any component of ${ }^{-}\left(\tilde{M}^{\prime}-N(k)\right)$. This implies that each arc of $\partial \tilde{M}^{\prime}$ that lies in the vertical part of $\partial F \times I$ is an excellent arc of intersection between $\bar{M}$ and $A$ or $\bar{M}$ and some $V_{i}$. Since there is at least one such arc we have a contradiction.

2. Least genus one-sided Heegaard splittings of the three-torus. The surface of nonorientable genus $h$, denoted $U_{h}$, is the connected sum of $h$ projective planes. Bredon and Wood [B-W], showed that if $F$ is a closed orientable surface of positive genus, then $U_{4}$ is the least genus nonorientable surface that embeds in $S^{\prime} \times F$. The three-torus possesses a one-sided Heegaard splitting of genus 4 . To see this, let $T$ be an incompressible torus in $S^{\prime} \times S^{\prime} \times S^{\prime}$. Notice that the three-torus cut along $T$ is isomorphic to $T \times I$. Let $k$ be an unknotted arc in $T \times I$ whose ends are not identified when $T \times I$ is glued back together to form the three-torus. Construct $U_{4}$ as follows. Let $N(k)$ be a small regular neighborhood of $k$ in $S^{\prime} \times S^{\prime} \times S^{\prime}$. Remove $N(k) \cap T$ from $T$ and replace it by the closure of the annulus component of $\partial N(k)-T$. From $\S 1$ we see that this copy of $U_{4}$ that we have constructed is a one-sided Heegaard splitting of the three-torus. Since $U_{4}$ is the least genus one-sided surface that embeds in the three-torus we have constructed a least genus one-sided Heegaard splitting of the three-torus. If we perform the same construction starting with an incompressible torus $T^{\prime}$ that is not $\mathbf{Z}_{2}$ homologous to $T$ we obtain a one-sided Heegaard splitting of the three-torus by $U_{4}$ that cannot be isotopic to our original one-sided Heegaard splitting. We can however show the following.

THEOREM 2.1. Least genus one-sided Heegaard splittings of the threetorus are unique up to homeomorphism.

Proof. We will show that if $K$ is a least genus Heegaard splitting of the three-torus then $K$ is obtained by surgering an incompressible torus $T \subseteq S^{\prime} \times S^{\prime} \times S^{\prime}$ along an arc that is unknotted in $T \times I$, the topological uniqueness of least genus one-sided Heegaard splittings of the three-torus will then be evident.

In $[\mathbf{F r}]$ it is shown that an orientable $S^{\prime}$-bundle over a closed orientable surface contains a one-sided incompressible surface if and only if it has even nonzero Euler class. Hence $K \subseteq S^{\prime} \times S^{\prime} \times S^{\prime}$ is compressible. Let $D$ be a compressing disk for $K$. Let $h: D \times[0,1] \rightarrow S^{\prime} \times S^{\prime} \times S^{\prime}$ 
be an embedding such that $\left.h\right|_{D \times\{1 / 2\}}\left(D \times\left\{\frac{1}{2}\right\}\right)=D$ and $h^{-1}(K)=\partial D$ $\times[0,1]$. Notice that $T=K-h(\bar{M} \partial D \times[0,1]) \cup h(D \times\{0,1\})$ must be orientable (Bredon and Wood) and nonseparating (surgering along a disk does not change the $\mathbf{Z}_{2}$ homology class). Hence $T$ is an incompressible torus. Furthermore $K$ is obtained by surgering $T$ along $h(* \times I)$, where * $\in$ int $D$. Since $S^{\prime} \times S^{\prime} \times S^{\prime}-K$ is a handlebody, we have that $S^{\prime} \times S^{\prime} \times S^{\prime}-K-h\left(D^{2} \times I\right)$ is a handlebody. By Lemma 1.1 we have that $h(* \times I)$ is unknotted in $T \times I$.

It is worth noting that if $K$ is a genus 4 one-sided Heegaard splitting of the three-torus $M$, then the double cover of the three-torus corresponding to the orientable double cover of $K$ is again a three-torus $\tilde{M}$ and the double cover $F$ of $K$ in $\tilde{M}$ is a genus three Heegaard splitting of the three-torus $\tilde{M}$. Theorem 2.1 implies the topological uniqueness of one-sided genus 4 minimal surfaces in a flat three-torus.

3. Genus three minimal surfaces in a flat three-torus. A flat threetorus is $S^{1} \times S^{1} \times S^{1}$ equipped with a Riemannian metric having all its sectional curvatures equal to zero. Given a flat three-torus $M$ the universal cover of $M$ can be realized geometrically as $\mathbf{R}^{3}$ so that the deck transformations are translations by the vectors in some cocompact lattice $\Gamma \subseteq \mathbf{R}^{3}$. Notice that $M$ inherits a group structure from a realization of its universal cover, just take the group structure on $\mathbf{R}^{3} / \Gamma$. Inversion $\phi$ in such a group structure can be lifted to $\tilde{\phi}: \mathbf{R}^{3} \rightarrow \mathbf{R}^{3}, \tilde{\phi}\left(x_{1}, x_{2}, x_{3}\right)=-\left(x_{1}, x_{2}, x_{3}\right)$. Meeks [M1, M2], shows that a minimal surface $F$ of negative Euler characteristic in a flat three-torus is a Heegaard splitting; furthermore if $F$ has genus three, after a suitable choice of the identity for a group structure on the three-torus, $\phi(F)=F$ and $\left.\phi\right|_{F}: F \rightarrow F$ is the hyperelliptic involution of the conformal structure on $F$ inherited from the three-torus. A hyperelliptic involution on a closed orientable surface $F_{g}$ is an involution that is the deck transformation of a branched cover $b: F_{g} \rightarrow S^{2}$. All hyperelliptic involutions on a given surface are topologically equivalent. If $\chi$ is a simple closed curve on $S^{2}$ that is disjoint from the branch set of $b$ then $\chi$ lifts to $F_{g}$ if and only if a component of $S^{2}-\chi$ contains an even number of branch points.

The quotient space of $S^{1} \times S^{1} \times S^{1}$ under the action of $\phi$ is a three-manifold with 8 singularities which are cones on $\mathbf{P}^{2}$. Denote by $Q$ the quotient space with the interiors of small regular neighborhoods of the singularities removed. The image of $F$ in $Q$ is a planar surface 0 with eight boundary components, each one a nontrivial curve in one of the 
eight boundary components of $Q$. In Lemma 3.4 we will show that 0 is a compressible surface, but for the sake of exposition we assume Lemma 3.4 for the time being and proceed.

THEOREM 3.1. Up to topological equivalence there is only one genus three surface $F$ in the three-torus that can be a minimal surface in a flat three-torus.

Proof. From [M1] we know that if $F$ is a minimal surface in some flat three-torus $M$ then $F$ is a Heegaard splitting of $M$. We will show that $F$ is a "standard" Heegaard splitting, which is enough to prove our result.

Let 0 and $Q$ be as above. By Lemma 3.4 the surface 0 is compressible in $Q$. Let $D$ be a compressing disk for 0 . Since $\partial D$ lifts to $F$ we have that $\partial D$ on $F / \phi\left(\cong S^{2}\right)$ must separate $F / \phi$ into two disks each containing an even number of branch points. This can be seen from the monodromy representation for the hyperelliptic involution. Let $D_{1}$ and $D_{2}$ be the lifts of $D$ to $M$. Notice that $D_{1}$ and $D_{2}$ lie on opposite sides of $F$. Suppose that one of the components $C$ of $F / \phi-D$ contained only two branch points, then $C$ lifts to an annulus $A$ on $F$ with $\partial A=\partial D_{1} \cup \partial D_{2}$. Notice that $D_{1} \cup A \cup D_{2}$ is a nonseparating sphere in $M$. This is absurd; hence we may assume that $\partial D$ separates $F / \phi$ into two components each containing 4 branch points. Thus $\partial D_{1}$ and $\partial D_{2}$ separate $F$ into two tori having two boundary components apiece. Let $h_{l}: D_{l} \times I \rightarrow M$ with $D_{i}=h\left(D_{i} \times\left\{\frac{1}{2}\right\}\right)$ be embeddings such that $h_{l}^{-1}(M)=\partial D_{l} \times I$ $\& h_{1}^{-1}\left(h_{2}\left(D_{2} \times I\right)\right)=\varnothing$. Surger $F$ by replacing $h_{1}\left(\partial D_{1} \times I\right)$ and $h_{2}\left(\partial D_{2} \times I\right)$ by $D_{1} \times\{0,1\}$ and $D_{2} \times\{0,1\}$. The result is two parallel nonseparating tori $T_{1}$ and $T_{2}$. Hence $T_{1}$ and $T_{2}$ separate $M$ into two copies of the cartesian product of a torus and an interval. Let $T_{i} \times I$ denote the copy of $S^{\prime} \times S^{\prime} \times I$ containing $D_{l}$. Furthermore if $k_{l}=$ $h_{i}(* \times I)$ with $* \in \operatorname{int} D_{i}$ then $F$ is obtained by surgering $T_{1} \cup T_{2}$ along $k_{1}$ and $k_{2}$. For $F$ to be a Heegaard splitting ${ }^{-}\left(T_{i} \times I-N\left(k_{i}\right)\right)$ must be a handlebody for $i=1,2$. By Lemma 1.1 the arc $k_{i}$ must be unknotted in $T_{i} \times I$. This is enough to show that $F$ is topologically unique.

REMARK. Using a good picture of $F$ and the fact that the mapping class group of the three-torus is generated by Dehn twists along appropriately chosen tori, we may in fact show that $F$ is unique up to isotopy.

It can be seen that if $G$ is the double cover of a one-sided Heegaard splitting of the three-torus by $U_{4}$ then $G$ is isotopic to the surfaces $F$ described above. 
It still remains to show that 0 is compressible. To do this we will assume that 0 is incompressible and isotope 0 into a "standard" position that will make the absurdity of our assumption obvious. Let $h: S^{1} \times S^{1}$ $\rightarrow S^{1} \times S^{1}$ be the hyperelliptic involution. If we view $S^{1}$ as $\mathbf{R} / \mathrm{Z}$ then $h\left(x_{1}, x_{2}\right)=-\left(x_{1}, x_{2}\right)$. Let $h_{0}$ and $h_{1}$ be the maps induced on $S^{1} \times S^{1} \times$ $\{0\}$ and $S^{1} \times S^{1} \times\{1\}$ via the obvious identification. Then $Q$ is homeomorphic to $S^{1} \times S^{1} \times I / h_{0}, h_{1}$ with the interior of small regular neighborhoods of the fixed points of $h_{0}$ and $h_{1}$ removed. Let $P_{0}$ and $P_{1}$ be the planar surfaces in $Q$ corresponding to $S^{1} \times S^{1} \times\{0\}$ and $S^{1} \times S^{1} \times\{1\}$ respectively.

\section{LEMMA 3.3. $Q$ is irreducible.}

Proof. Realize the universal cover $\tilde{Q}$ of $Q$ as $\mathbf{R}^{3}$ with small open balls removed from around the points of the form $\frac{1}{2}(a, b, c)$ with $a, b, c \in \mathbf{Z}$, so that the group of deck transformations is generated by the integral translations and the map $\tilde{\phi}\left(x_{1}, x_{2}, x_{3}\right)=-\left(x_{1}, x_{2}, x_{3}\right)$. (Note that this realization is only topological. Incidentally the surfaces $P_{0}$ and $P_{1}$ can be chosen to be the images of $x_{3}=0$ and $x_{3}=\frac{1}{2}$ in $Q$.) Let $S$ be a sphere embedded in $Q$. Let $\tilde{S}$ be a lift of $S$ to $\tilde{Q}$. Notice that since $S$ is embedded if $d \tilde{S} \cap \tilde{S}=\varnothing$ for some deck transformation then $d$ is the identity. Since $\tilde{Q} \subseteq \mathbf{R}^{3}$ we have that $\tilde{S}$ bounds a punctured ball $\tilde{B}$ in $\tilde{Q}$. If the ball is genuinely punctured then there is a nontrivial deck transformation preserving a puncture of $\tilde{B}$ and consequently either $d \tilde{B} \subseteq \tilde{B}$ or $\tilde{B} \subseteq d \tilde{B}$. Since $d$ is nontrivial the valid inclusion is strict, but then $d$ does not preserve volume on $\mathbf{R}^{3}$. This contradicts our choice of the universal cover of $Q$. Therefore $\tilde{B}$ is a ball and $S$ bounds the ball in $Q$ that is the image of $\tilde{B}$.

\section{LEMMA 3.4. The surface 0 is compressible.}

Proof. Assume that 0 is incompressible. Isotope 0 so that it is transverse to $P_{0}$ and $P_{1}$ and $0 \cap\left(P_{0} \cup P_{1}\right)$ has the smallest number of components possible. Since both $\partial 0$ and $\partial\left(P_{0} \cup P_{1}\right)$ consist of a single nontrivial curve on each boundary component of $Q$, we can isotope 0 without increasing the number of components of $0 \cap\left(P_{0} \cup P_{1}\right)$ so that each boundary curve of 0 intersects $\partial\left(P_{0} \cup P_{1}\right)$ exactly once in a point of transverse intersection. Hence $0 \cap P_{i}$ contains exactly two arcs $k_{1 i}, k_{2 i}$ and they link distinct components of $\partial P_{i}$. Let $\bar{Q}$ denote $Q$ cut along $P_{0}$ and $P_{1}$. Since $0 \cap\left(P_{0} \cup P_{1}\right)$ is minimal the image $\overline{0}$ of 0 in $\bar{Q}$ is 
incompressible. Since $Q$ is irreducible and $0 \cap\left(P_{0} \cup P_{1}\right)$ is minimal we have that there are no curves in $0 \cap P_{i}$ that are trivial on $P_{i}$. Hence $\partial \overline{0}$ consists of nontrivial simple closed curves on $\partial \bar{Q}$. From the choice of $P_{0}$ and $P_{1}$ we have that $\bar{Q}$ is homeomorphic to the cartesian product of a torus and the unit interval. Since $\overline{0}$ is incompressible, and $\partial \overline{0}$ consists of nontrivial simple closed curves, a result of Waldhausen [W3] implies that $\overline{0}$ is a family of annuli. Hence the Euler characteristic of $\overline{0}$ is zero. Since 0 is obtained from $\overline{0}$ by identifying 4 pairs of arcs in $\partial \overline{0}$, and some pairs of circles, we have that the Euler characteristic of 0 is -4 . Since 0 is a connected planar surface with eight boundary components, it has Euler characteristic -6 . This is a contradiction.

\section{REFERENCES}

[B] E. M. Brown, Unknotting in $M^{2} \times I$, Trans. Amer. Math. Soc., 123 (1966), 480-505.

[Fe] C. D. Feustal, Isotopic unknotting in $F \times I$, Trans. Amer. Math. Soc., 179 (1973), 227-238.

[Fr] C. D. Frohman, One-sided incompressible surfaces in Seifert fibered spaces, to appear in Topology Appls.

[L] H. B. Lawson, The unknottedness of minimal embeddings, Invent. Math., 11 (1970), 183-187.

[M1] W. H. Meeks, Lectures on Plateu's problem, IMPA, 1978.

[M2] The geometry and conformal structure of triply periodic minimal surfaces in 3, Doctoral Thesis, U. C. Berkeley, 1975.

[W1] F. Waldhausen, Heegaard Zerlegungen der 3-sphere, Topology, 7 (1968), 195-203.

[W2] __ Eine Klasse von 3-deminsionale Mannigfaltigkeiten I, Invent. Math., 3 (1967), 308-333.

[W3] On irreducible 3-manifolds that are sufficiently large, Ann. of Math., 87 (1968), 56-58.

Received April 20, 1985.

UNIVERSITY OF CALIFORNIA

SANTA Barbara, CA 97106 


\section{PACIFIC JOURNAL OF MATHEMATICS EDITORS}

\author{
V. S. VARADARAJAN \\ (Managing Editor) \\ University of California \\ Los Angeles, CA 90024 \\ HeRbert Clemens \\ University of Utah \\ Salt Lake City, UT 84112 \\ R. FINN \\ Stanford University \\ Stanford, CA 94305
}

HERMANN FLASCHKA

University of Arizona

Tucson, AZ 85721

RAMESH A. GANGOLLI

University of Washington

Seattle, WA 98195

VAUGHAN F. R. JONES

University of California

Berkeley, CA 94720

ROBION KIRBY

University of California

Berkeley, CA 94720
C. C. MOORE

University of California

Berkeley, CA 94720

H. SAMELSON

Stanford University

Stanford, CA 94305

HAROLD STARK

University of California, San Diego

La Jolla, CA 92093

\section{ASSOCIATE EDITORS}
R. Arens
E. F. BECKENBACH
B. H. NEUMANN
F. WOLF
K. YoshIDA (1906-1982)

\section{SUPPORTING INSTITUTIONS}

UNIVERSITY OF ARIZONA

UNIVERSITY OF BRITISH COLUMBIA

CALIFORNIA INSTITUTE OF TECHNOLOGY

UNIVERSITY OF CALIFORNIA

MONTANA STATE UNIVERSITY

UNIVERSITY OF NEVADA, RENO

NEW MEXICO STATE UNIVERSITY

OREGON STATE UNIVERSITY
UNIVERSITY OF OREGON

UNIVERSITY OF SOUTHERN CALIFORNIA

TANFORD UNIVERSITY

UNIVERSITY OF HAWAII

UNIVERSITY OF TOKYO

UNIVERSITY OF UTAH

WASHINGTON STATE UNIVERSITY

UNIVERSITY OF WASHINGTON 


\section{Pacific Journal of Mathematics}

Vol. 124, No. $1 \quad$ May, 1986

Kinetsu Abe and Martin Andrew Magid, Relative nullity foliations and indefinite isometric immersions

Erik P. van den Ban, A convexity theorem for semisimple symmetric

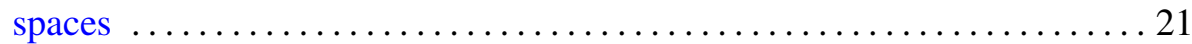

Bo Berndtsson and Thomas Joseph Ransford, Analytic multifunctions, the $\bar{\partial}$-equation, and a proof of the corona theorem .................. 57

Brian Boe and David H. Collingwood, Intertwining operators between

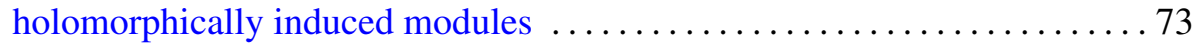

Giuseppe Ceresa and Alessandro Verra, The Abel-Jacobi isomorphism for

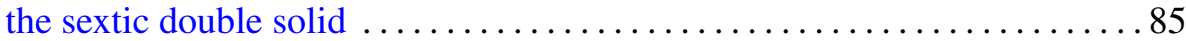

Kun Soo Chang, Jae Moon Ahn and Joo Sup Chang, An evaluation of the conditional Yeh-Wiener integral ........................... 107

Charles Dale Frohman, Minimal surfaces and Heegaard splittings of the

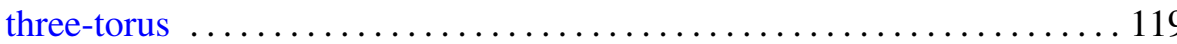

Robert M. Guralnick, Power cancellation of modules $\ldots \ldots \ldots \ldots \ldots \ldots 131$

Kenneth Hardy and Kenneth S. Williams, On the solvability of the Diophantine equation $d V^{2}-2 e V W-d W^{2}=1 \ldots \ldots \ldots \ldots \ldots \ldots \ldots \ldots \ldots$

Ray Alden Kunze and Stephen Scheinberg, Alternative algebras having

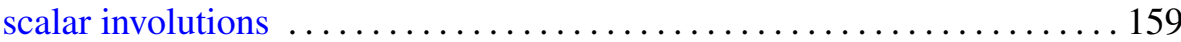

W. B. Raymond Lickorish and Kenneth Millett, The reversing result for the Jones polynomial $\ldots \ldots \ldots \ldots \ldots \ldots \ldots \ldots \ldots$

Guido Lupacciolu, A theorem on holomorphic extension of CR-functions

William Schumacher Massey and Lorenzo Traldi, On a conjecture of K. Murasugi

Dinakar Ramakrishnan, Spectral decomposition of $L^{2}(N \backslash \mathrm{GL}(2), \eta)$

Steven L. Sperber, On solutions of differential equations which satisfy certain algebraic relations 